\title{
On the significance of quantum effects and interactions for the apparent universality of Bloch laws for $M_{s}(T)$
}

\author{
U. Krey* \\ Inst. of Physics II, Universität Regensburg, D-09040 Regensburg, Germany
}

v1: March 12, v2=v3: May 27, 2003; accepted by JMMM

\begin{abstract}
The apparent universality of Bloch's $T^{3 / 2}$-law for the temperature dependence of the spontaneous magnetisation, and of generalizations thereof, is considered. It is argued that in the derivation one should not only consider the exchange interaction between the spins, but also the other interactions between them, leading to elliptical spin precession and deviations from the parabolic dispersion of magnons. Also interaction effects are important to explain the apparent universality of generalized Bloch law exponents $\epsilon_{B}$, defined by $M_{s}(T)=M_{s}(0)$-const. $T^{\epsilon_{B}}$, valid in a wide temperature range $T_{1}<T<T_{2}$, and for dimensionalities $d=1,2$, and 3 . The above-mentioned temperature range, the 'Bloch range', lies above the quantum range, where magnetic long-range order (e.g. in $d=2$ dimensions) is nontrivially enforced by the additional interactions, but below the thermal critical region, where universal 'anomalous scaling dimensions' apply. In contrast, for the Bloch temperature region, the universality is only apparent, i.e. a crossoverphenomenon, and simple scaling considerations with 'normal dimensions' apply. However, due to interactions, the Bloch exponent $\epsilon_{B}$ depends not only on the dimensionality $d$ of the system, but also on the spin quantum number $s(\bmod (1 / 2))$ of the system, i.e. for given $d$ the Bloch exponent $\epsilon_{B}$ is different for half-integer $s$ and for integer $s$.
\end{abstract}

PACS numbers: 75 . Magnetic properties;

05.50 Fh Phase Transitions: General Studies ;

*e-mail uwe.krey@physik.uni-regensburg.de 
Keywords: Bloch's law; Quantum Effects; Universality; Magnon Interactions

\section{Introduction:}

This paper grew out of discussions following a recent presentation of certain experimental results in our institute, which apparently, and unexpectedly, showed the $T^{3 / 2}$ Bloch law for $M_{s}(T)$ for a nanostructured planar system, [1]; and although I originally thought that the considerations presented below were too simple for publication, some of the participants of those discussions suggested that I should write them down. So here I do so, just hoping to broaden and intensify thereby more personal discussions at the expense of being necessarily rather informal.

Bloch's famous $T^{3 / 2}$-law, and the natural generalization thereof, is written as follows:

$$
M_{s}(T)=M_{s}(0)-\text { const. } \cdot T^{\epsilon_{B}}
$$

where according to the original derivation of $\mathrm{F}$. Bloch for a $d=3$ dimensional Heisenberg model, [2], the Bloch exponent $\epsilon_{B}$ would be $3 / 2$, while a recent analysis of various experimental data in different dimensions by U. Köbler and coworkers, see Ref. [3] and references therein, led to a set of values to be presented below, which are partially much different from the value $3 / 2$, but appear to be quite universal. In particular, the number $\epsilon_{B}=3 / 2$ does not only follow from the original theoretical analysis of $\mathrm{F}$. Bloch and the later refinement of this analysis by F.J. Dyson, [4], but (unexpectedly) it was also observed by $\mathrm{U}$. Gradmann et al. in $d=2$-dimensional ultrathin ferromagnetic films, see e.g. Ref. [5], and now it is apparently also observed in the planar nanostructures of Ref. [1]. Moreover, the experimental analysis of U. Köbler et al., Ref. [3], led to the above-mentioned set of apparently universal numbers for the Bloch exponent $\epsilon_{B}$, where $\epsilon_{B}$ depends not only on the dimensionality $d$ of the ferromagnetic or antiferromagnetic system considered, but also assumes different values for integer rsp. half-integer spin quantum number s: namely $\epsilon_{B}=5 / 2$ rsp. $=3 / 2$ rsp. 2 for $d=1$ rsp. $=2$ rsp. 3 and half-integer s; whereas $\epsilon_{B}=3$ rsp. $=2 \mathrm{rsp} .9 / 2$ for $d=1 \mathrm{rsp} .=2 \mathrm{rsp} .3$ and integer $\mathrm{s}$. (The $\epsilon_{B^{-} \text {values }}$ 
for $d=1 \mathrm{rsp} .=2$ are also observed for anisotropically disordered or amorphous $d=2$ - rsp. = 3-dimensional systems.)

Of course, as a theorist, one should always be skeptical against experimental data which may be biased by theoretical claims. Therefore, in the present context it is stressed right from the beginning that the following presentation is not based on these data, but only motivated by it.

In particular, and intentionally, I always write 'apparently universal' instead of simply 'universal', since the Bloch behaviour of Eqn. (1) is generally neither observed in the region of extremely low temperatures near $T=0$, where quantum behaviour dominates, nor is it observed in the genuine thermal critical region, where one would obtain scaling with so-called anomalous dimensions, i.e. the 'critical exponents'. Instead, the above-mentioned behaviour should be considered as a crossoverphenomenon, observed in a (wide) intermediate temperature region $T_{1}<T<T_{2}$, see below. In particular, the scaling dimensions involved correspond to simple powers of the basic thermal energy $k_{B} T$ rsp. of $\left(k_{B} T\right)^{1 / 2}$, where $k_{B}$ is Boltzmann's constant. But in agreement with the fact that magnon-magnon interactions (although not yet thermally critical) already plays a role in the Bloch region (particularly at $d=2$ ), the Bloch exponent, according to the considerations presented below, depends on the spin quantum number $s$ in the above-mentioned way.

The schedule of the present paper is as follows: In the next chapter the original Bloch derivation for a $d=3$-dimensional Heisenberg system is considered, and, for later heuristical purposes, a simplified 'poor man's' variant thereof. Then I consider planar systems $(d=2)$. Next, $d=1$ and $d=3$ follow, all three cases for $s=1 / 2$. Finally I consider $s=1$, thereby collecting the whole set of 'apparent Bloch universality classes' of Köbler. At the end, in an additional chapter, the conclusions are presented.

\section{Bloch's law for $d=3$}

According to this law (which is derived in any textbook on solid-state magnetism and is one of the most prominent results of theoretical physics) the temperature dependence of the magnetisation $M_{s}(T)$ of 
a three-dimensional ferromagnet is given by

$$
M_{s}(T) \equiv M_{s}(0)-\text { const. } \times \int_{B Z} \mathrm{~d}^{3} k \frac{1}{\exp \beta \epsilon(\vec{k})-1},
$$

because each excited magnon reduces the magnetic moment of a ferromagnetic sample by a Bohr magneton. Here $T$ is the Kelvin temperature, $\beta=\left(k_{B} T\right)^{-1}$ with the Boltzmann constant $k_{B}$, and the fraction $\{\exp (\beta \epsilon(\vec{k}))-1\}^{-1}$ represents the thermal expectation value of the number of magnons with excitation energy $\epsilon(\vec{k})$, where the wave-vector $\vec{k}$ has its usual meaning. The integration is over the Brillouin zone BZ of the crystal. Since for $k \cdot a \ll 1$ (where $a$ is the lattice constant) the excitation energy has a parabolic dispersion, $\epsilon(\vec{k}) \propto k^{2}$, the integral in Eq. (2) converges for $d=3$, whereas for $d=2$ it would be 'infrared divergent' in agreement with the famous Mermin-Wagner theorem, [6].

For the excitation energy $\epsilon(\vec{k})$ of a magnon in a Heisenberg ferromagnet (and also in itinerant ferromagnets when the spin-orbit interaction, and also the Stoner excitations, are neglected against the collective magnon-like spin excitations) one simply has the following dispersion (we assume cubic symmetry and extend the validity of the formula over the whole integration region): $\epsilon(\vec{k})=D \cdot k^{2}$, where $D$ is the so-called spin-wave stiffness. Therefore, by the substitution $x:=\beta D \cdot k^{2}$ and the replacement $\mathrm{d}^{3} k=4 \pi k^{2} \mathrm{~d} k$ one gets the famous result $M_{s}(T)=M_{s}(0)-$ const $^{\prime} \cdot \cdot\left(\frac{k_{B} T}{D}\right)^{3 / 2}$, where (up to exponentiallysmall terms) the constants const. and const.' are related as follows: const.' = const. $\times 2 \pi \int_{0}^{\infty} \frac{x^{1 / 2} \mathrm{~d} x}{\exp x-1}$. The analysis has been refined in the monumental work of F.J. Dyson, see Ref. [4], but this is not important below, except at the end.

Rather, for Anderson's "poor man", [7], instead of the usual derivation, one can give the following simpler argument, which later-on will also serve for heuristical purposes: $\exp \beta D k^{2}-1$ is approximated for long enough wavelengths and/or high enough $T$ by the 'quasi-classical thermal-energy approximation' $\beta D \cdot k^{2}$, so that one simply gets $M_{s}(T)=$ $M_{s}(0)-$ const. $\left(\frac{k_{B} T}{D}\right) \times P h s R(T)$, where the factor $k_{B} T$ represents the thermal equipartition energy, while $\operatorname{Phs} R(T)$ means a typical phase- 
space radius in $\mathrm{k}$-space ${ }^{1}$, replacing the integral $\int_{0}^{\prime \pi / a^{\prime}} \frac{4 \pi k^{2}}{k^{2}} \mathrm{~d} k \times g_{k}(T)$, where $g_{k}(T)$ is a temperature-dependent dimensionless weight-function. Here ${ }^{\prime} \pi / a^{\prime}$ represents a (very large) wavenumber-cutoff corresponding to the upper edge of the Brillouin zone, which is replaced by a sphere as in typical renormalization group arguments. But it would be wrong, if at this place one would perform directly the integration without any idea of the weight-function (after having made the above-mentioned 'equipartition approximation' leading to the 'quasi-classical thermalenergy prefactor' $\left(\frac{k_{B} T}{D}\right)$ ). Instead, one gets the correct $P h s R(T)$ by a simple 'Pippard argument', i.e. simply by equating the dominating energy- resp. temperature-ranges: $\epsilon\left(\vec{k}_{\text {dom. }}\right)=D \cdot k_{\text {dom. }}^{2} \equiv k_{B} T$, i.e. $\operatorname{PhsR}(T) \equiv k_{\text {dom. }}(T)(\cong \Delta k(T)) \equiv\left(\frac{k_{B} T}{D}\right)^{1 / 2}$.

\section{Planar systems}

What would be different in a planar system magnetised in-plane ?

One would again expect a quasi-classical 'equipartition approximation' $\exp \beta \epsilon(\vec{k})-1 \rightarrow \beta \epsilon(\vec{k})$, but otherwise one would have a lot of differences and, generally, quite subtle behaviour:

- First, instead of the simple parabolic dispersion $\epsilon(\vec{k})=D \cdot k^{2}$, representing excitations with circular spin precession, one would have a more general 'square root' formula, $\epsilon(\vec{k})=\sqrt{\epsilon_{a}(\vec{k}) \cdot \epsilon_{b}(\vec{k})}$, representing an elliptical precession with the main axes $a$ and $b$, respectively.

For example, if one is dealing with a film of infinite extension in the $\mathrm{x}$ - and $\mathrm{y}$-directions, with almost neglegible thickness $t_{h}$, then (if the film is magnetised in-plane, e.g. in the x-direction, see below) spin-wave deviations in the z-direction are strongly disfavoured due to the demagnetising field $H_{z}^{\mathrm{DM}}=-4 \pi M_{z}$, where $H_{z}^{\mathrm{DM}}$ and $M_{z}$ are the $\mathrm{z}$-components of the demagnetising field and the magnetisation, respectively. Thus, one would have $\epsilon_{b}(\vec{k}) \equiv$ $C_{b}+D \cdot k^{2}\left(\cong C_{b}\right.$ in the long-wavelength limit, where $C_{b}=$ $\mu_{B} \cdot 4 \pi M_{s}$ is the effective anisotropy energy corresponding to the

\footnotetext{
${ }^{1}$ i.e. PhsR(T) is some kind of reciprocal thermal de Broglie wavelength
} 
demagnetising field $\left(\mu_{B}\right.$ is Bohr's elementary magnetic moment, the 'magneton'.)). In contrast, spin deviations in the $y$-direction would not be disfavoured, i.e. $\epsilon_{a}(\vec{k}) \cong D \cdot k^{2}$, as before (but see the following text, which should make things more precise):

Actually, the magnetostatic interactions giving rise to the abovementioned demagnetising field $-4 \pi M_{z}$, also produce in-plane field components, which are proportional to the (small) thickness $t_{h}$ of the ultrathin film. (Here, 'ultrathin' means that $t_{h}$ is of the orderof-magnitude of some lattice constants; thus, even a monolayer should be approximated in this way by a continuous film of finite thickness $t_{h}=a$, and one should not simply set $t_{h}=0$, instead.)

- Second, the in-plane magnetostatic fields are not constant, but in contrast to the exchange interactions and to the usual singleion anisotropies, they are long-ranged and induce a finite in-plane value of the magnetisation, although for the present planar symmetry the Mermin-Wagner theorem, see Ref. [6], in the presence of only short-range interactions would rigorously predict vanishing long-range order. Actually, the above-mentioned finite magnetisation is not in contradiction with this theorem nor with a recent extension, [8], since in these papers the magnetostatic interaction is not included.

- Third, generically, by the magnetostatic fields and/or other interactions, one has an effective out-of-plane anisotropy, favouring

1. an out-of-plane state of the magnetisation (this case will be considered later), or

2. an in-plane state, a case, on which we concentrate first:

In the above-mentioned situation, for vanishing thickness, i.e. in the formal limit $t_{h} \rightarrow 0$, this in-plane state would be described by a model of short-range XY-symmetry, which has a phase transition to a low-temperature phase with diverging susceptibility, but without long-range order for any finite $T$, the 'Kosterlitz-Thouless transition' (KT transition), see Ref. [9]. But for finite thickness, according to an equation to be presented below, there is always a $\vec{k}$-dependent long-range effective in-plane uniaxial anisotropy 
favouring e.g. a spin-alignment along the (arbitrary) $\pm x$-direction and disfavouring spin fluctuations with finite in-plane $\vec{k}$ along the $\pm y$-axis, i.e. perpendicular to the magnetisation. (E.g. according to Hoffmann's well-known 'ripple theory', [10], the so-called 'transverse ripple', with $\vec{k}= \pm(0, k, 0)$, is disfavoured with respect to the longitudinal one, where $\vec{k}= \pm(k, 0,0)$, see also Ref. [11].)

- In fact, if the magnitude $k$ of the (in-plane) wavevector $\vec{k}$ times the thickness $t_{h}$ of the film is sufficiently low, due to long-range magnetostatic interactions, for in-plane magnetised thin films with purely dipolar and exchange interactions, the magnon energy $\epsilon(\vec{k})$ is given by the following formula, see Ref. [12], Eqs. (11)-(14):

$$
\frac{\epsilon(\vec{k})}{\mu_{B}}=\sqrt{\left(H_{x}+\frac{2 A}{M_{s}} k^{2}\right) \cdot\left(H_{x}+4 \pi M_{s}+\frac{2 A}{M_{s}} k^{2}\right)+\frac{k \cdot t_{h}}{2}\left(4 \pi M_{s}\right)^{2}\left(\frac{k_{y}^{2}}{k^{2}}\right)+\ldots,}
$$

where the dots denote neglected terms $\propto t_{h}^{2}$ or higher powers of $t_{h}$; i.e. to avoid z-dependencies it is assumed in the following that the thickness $t_{h}$ of the film, although $>0$, is nonetheless much smaller than the magnetostatic exchange length $l_{e}$ defined below. Furthermore, $\mu_{B}$ is the Bohr 'magneton', $\mu_{B} \cdot \frac{2 A}{M_{s}} \equiv D$ the spin-wave stiffness, $M_{s}$ the saturation magnetisation in c.g.s. units, and $H_{x}$ a small external field in the $x$-direction. (As mentioned above, for $t_{h}=0$ this Zeeman field would be necessary to break the $X Y$-symmetry which for short-range interactions would imply vanishing long-range order. Actually, the same magnetisation divergence giving rise to the term $\propto k_{y}^{2} / k^{2}$ in Eqn. (3) (note the non-analytic behaviour on the direction!) would also give a contribution $\propto k_{y} k_{x} / k^{2}$ modifying $H_{x}$ : this is usually neglected. However, in principle the neglection is not allowed for small $H_{x}$; e.g. the additional terms give rise to a 'blocking phenomenon' of the magnetisation ripple, see Ref. [10]. But here we neglect this addition as usual, since we do not consider that phenomenon.)

- At the same time, due to the fact that by the strong ellipticity of the spin-precession the in-plane amplitudes are extraordinarily large compared with the out-of plane amplitudes, which reduces the expectation value of the magnetisation much more 
than in case of circular precession, the integrand on the r.h.s. of Eqn. (2) is multiplied by an additional 'ellipticity factor' $f(\vec{k})=$ $\frac{\left(\epsilon_{a}(\vec{k})+\epsilon_{b}(\vec{k})\right) / 2}{\sqrt{\epsilon_{a}(\vec{k}) \cdot \epsilon_{b}(\vec{k})}}$, which is always $\geq 1$; i.e. now one would get instead of Eqn. (2):

$$
M_{s}(T)=M_{s}(0)-\text { const. } \times \int_{0}^{\prime \pi / a^{\prime}} \mathrm{d}^{2} k \frac{1}{\exp (\beta \epsilon(\vec{k}))-1} \cdot f(\vec{k}) .
$$

(Below, this still seemingly harmless and natural-looking equation, which is obtained by a standard Bogoliubov-Valatin transformation, see e.g. p. 25 of [13], from the bilinearized (i.e. approximate!) spin Hamiltonian of the system, will be modified by spin interactions. This leads to a further temperature dependent phenomenological 'interaction factor' $F_{k}(T)$, which is important in the Bloch region of $d=2$-dimensional systems.)

If one would again consider the formal limit $t_{h} \rightarrow 0$ of the Eqs. (3) and (4), then the integral on the r.h.s. of Eq. (4) would diverge, although the fraction in front of $f(\vec{k})$ is now no longer dangerous. But the 'ellipticity factor' $f(\vec{k})$ itself diverges: $f(\vec{k}) \rightarrow k^{-1}$ for $k \cdot t_{h} \rightarrow 0$, and this ensures the logarithmic divergency of the integral in Eqn. (4). In fact, this divergency reflects the short-range XY-symmetry in the limit $t_{h} \equiv 0$ and is in agreement with the Mermin-Wagner theorem, see [6], since now all interactions have become short-ranged. But since for finite, but very small thickness, one has $\epsilon(\vec{k}) \propto\left(k \cdot t_{h}\right)^{1 / 2}\left|\frac{k_{y}^{2}}{k^{2}}\right|^{1 / 2}$, a careful integration shows not only that the integral (4) converges (the dangerous part is $\propto \int_{k=0}^{\prime \pi / a^{\prime}} \int_{\phi=0}^{2 \pi} \frac{k \mathrm{~d} k \mathrm{~d} \phi_{k}}{\left(k \cdot t_{h}\right)\left(4 \pi M_{s}\right) \sin ^{2} \phi_{k}+\frac{4 A}{M_{s}} k^{2}}$, which is actually very subtle since both $t_{h}$ and also $A / M_{s}$ must be different from 0 ), but according to [14] one also gets a $T^{3 / 2}$-behaviour at very low temperatures. However this is not relevant, since according to [14] this $T^{3 / 2}$-behaviour is only observed in the lowest temperature-range, where the dipolar coupling is all-important, e.g. $0 \leq T<T_{M} \cdot\left(\frac{t_{h}}{l_{e}}\right)$, where $T_{M}=\mu_{B} 4 \pi M_{s} / k_{B}$ is a characteristic temperature for the dipolar 'shape anisotropy', while $l_{e}=\sqrt{\frac{2 A}{2 \pi M_{s}^{2}}}$ is the already-mentioned 'magnetic exchange length', [15]. 
In Permalloy, with $4 \pi M_{s}=10^{4}$ Gauss and $A=1.310^{-6} \mathrm{erg} / \mathrm{cm}$ (remember $C_{b}=\mu_{B} 4 \pi M_{s}$ and $D:=\mu_{B}\left(2 A / M_{s}\right)$ ), the temperature $T_{M}$ would correspond to $0.67 \mathrm{~K}$. As mentioned above, the temperature region for the Bloch behaviour is, instead, rather high: $T_{1}<T<T_{2}$. Estimates for the two crossover temperatures $T_{1}$ and $T_{2}$ are:

- $T_{1} \cong \sqrt{2} \cdot \mu_{B} 4 \pi M / k_{B}$; this is slightly larger than $T_{M}$, but still very low compared to the temperature corresponding to the maximal spin-wave frequency, $T_{\max }: \cong D \cdot\left(\frac{\pi}{a}\right)^{2} / k_{B}(\cong 845 \mathrm{~K}$, with a lattice constant of $a \cong \pi 10^{-8} \mathrm{~cm}$, say).

- But it is known that Bloch's $T^{3 / 2}$-law applies to very high temperatures, e.g. up to $T_{2} \approx T_{\max } / 3$, without significant corrections, in planar systems of the above-mentioned kind, [5, 3]. And it should be stressed again at this place that this wide temperature region (the 'Bloch region') is still below the thermal critical region, [16].

In contrast, the relation $\epsilon(\vec{k}) \propto\left(k \cdot t_{h}\right)^{1 / 2}\left|\frac{k_{y}^{2}}{k^{2}}\right|^{1 / 2}$ is only valid for $k \lesssim k_{c}$, where $k_{c}:=t_{h} / l_{e}^{2}$ is a small 'crossover value': For larger wavenumbers the exchange interaction starts to dominate, i.e. $f(\vec{k}) \rightarrow$ 1 , and

$$
M_{s}(T) \cong M_{s}(\tilde{T})-\text { const. } \times \int_{k_{c}}^{\prime \pi / a^{\prime}} 2 \pi k \mathrm{~d} k \frac{1}{\exp \left(\beta D \cdot k^{2}\right)-1}
$$

where $k_{B} \tilde{T}:=\epsilon\left(k_{c}\right)$. By numerical evaluation (see below) this would inevitably lead to some kind of approximate 'Döring behaviour', [17], i.e. to $M_{s}(T)=M_{s}(\tilde{T})-$ const. ${ }^{\prime \prime \prime} \times T \cdot \ln \frac{T}{\tilde{T}}$, where $\tilde{T}$ can be considered as a temperature unit entering e.g. by quantum effects enforcing longrange order (In fact it turns out below that $\tilde{T}$ is identical with $T_{1}$ ). As far as the author knows, the 'Döring behaviour' has never been observed, although, in the temperature range considered, it was also suggested by Maleev, [14], in a paper preceding that of Bruno, [18].

- Instead, to get the observed $T^{3 / 2}$-behaviour (see $\left.[5,3]\right)$ also in the 'Döring temperature range', i.e. also for $T \gtrsim T_{1}$, one should note that, as soon as the wave-number $k$ is large enough, there is no longer any in-plane uniaxial anisotropy felt by the magnons. 
This means that it is natural to assume, as in the $d=2$-dimensional $\mathrm{XY}$ model, that there exists a strong enhancement of the destructive character of these magnetic excitations, 'destructive' with respect to the magnetic long range order, leading to an additional temperaturedependent factor $F_{k}(T)$ which represents the interaction of the magnetic quasiparticle excitations. In the planar XY model, this additional 'destructive factor' acts on all $k$-scales ( $T$-scales) until $k=0(T=0)$; therefore, the magnetic long range order is completely destroyed in the XY-model. Physically, this happens through the dynamic formation of magnetic vortex excitations: In the low-temperature phase of the KT transition these vortices are strongly bound in pairs of opposite topological charge (opposite chirality), and the typical distance of the vortex centers of such 'vortex pairs', $\langle l\rangle_{T}$, remains finite, if the critical temperature is attained: At this temperature there is vortex unbinding at $\langle l\rangle_{T_{c}}=l_{V}$.

- As a consequence, spinwaves are not influenced by the vortex formation, if their wavelength $\lambda=2 \pi / k$ is much larger than $l_{V}$, whereas spatial spin-spin correlations with distances $\underline{\text { much }}$ smaller than $l_{V}$ are wiped out by the vortices.

Similarly, we assume that in the Bloch region there is for $k>$ $k_{c}$ a 'reduction factor' $F_{k}(T)$ representing the enhanced reduction of magnetic order by the interactions between magnetic excitations of wavenumber $\vec{k}$. In the present case, this reduction factor is not as fatal as in the $d=2-\mathrm{XY}$ model, since wavenumbers smaller than $k_{c}$ are not involved. But one has again some kind of Pippard heuristics: Namely, along the lines of the original arguments of Kosterlitz, [19], we assume that $F_{k}(T) \propto\langle k\rangle_{T} \cdot l_{V}$, where $\langle\ldots\rangle_{T}$ denotes a thermal average, so that the reduction increases with increasing $T$, i.e. increasing $\langle k\rangle_{T}$. In fact, according to the (by now already known) Pippard argument, $\langle k\rangle_{T}$ can be replaced by $\left(k_{B} T / D\right)^{1 / 2}$. Thus again $M_{s}(T)=M_{s}(\tilde{T})-$ const. $\times T \times T^{1 / 2}$, where on the r.h.s. the factor $T$ represents the 'equipartition energy', while (for $d=2$ ) the factor $T^{1 / 2}$ arises from the 'reduction factor': This factor represents the strong reduction of long-range order by spin interaction effects, which are strongly 'relevant' in $d=2$. (In $d=3$ dimensions the 'reduction by interaction' is apparently not so important in the Bloch region. There, 
instead, the magnetic moment of whole columns of sites with identical planar coordinates $(\mathrm{x}, \mathrm{y})$ is reduced by the additional fluctuations corresponding to dynamic noncollinearities of the spins in the $z$-direction. This effect for $d=3$ is already described with noninteracting magnons by our previous factor PhsR(T). Thus, $d=2$ is much more dangerous to interactions than $d=3$, in agreement with the fact that the 3-dimensional $X Y$-model is 'harmless' compared with the $d=2$ dimensional $X Y$-problem.)

There is another argument concerning $F_{k}(T) \propto T^{1 / 2}$ : Since the typical thermal energy of an entity representing one degree of freedom is $k_{B} T$, and since the interaction should be dominated by two-body scattering, one guesses that in systems with strong magnon-magnon interactions the quasiparticle numbers $\left\langle n_{k}\right\rangle_{T}=\left\{\exp (\beta \epsilon(\vec{k})-1\}^{-1}\right.$ should be multiplied in the present context by $\left(k_{B} T\right)^{1 / 2}$ : This concerns the first particle of an interacting pair. A similar interaction factor $\left(k_{B} T\right)^{1 / 2}$ should come from the second particle; i.e. together the two interacting particles might be considered as an entity involving the genuine thermal energy $k_{B} T=\left[\left(k_{B} T\right)^{1 / 2}\right]^{2}$. This is actually again some kind of Pippard argument; it will be repeated later, when the transition from $s=1 / 2$ to $s=1$ is considered in section 8 .

\section{Quantum effects and crossover-flow for $\mathrm{d}=2$}

It is important to understand what is going on: Therefore in the following we sketch a flow-line scenario for an in-plane magnetised thin film. Generally, we consider in the following a cartesian coordinate system where on the abscissa axis (' $x$-axis') we plot the effective out-of-plane anisotropy energy $C_{b}$ (e.g. $\left.C_{b}=\mu_{B} 4 \pi M_{s}\right)$, which favours an in-plane magnetisation and is at the same time proportional to an effective uniaxial anisotropy energy (e.g. $\epsilon(\vec{k}) \cong \mu_{B} 4 \pi M_{s}\left[\left(k \cdot t_{h} / 2\right) \sin ^{2} \varphi_{k}\right]^{1 / 2}$ ) favouring an (arbitrary) $\pm x$-direction as in-plane magnetisation direction.

On the ordinate (' $y$ '-axis) of our plot the reduced Kelvin temperature $k_{B} T$ is presented. The origin of the cartesian coordinate system is at $\left(C_{g}=0, T=0\right)$.

On the third axis (' $z$-axis) the magnetisation $M_{s}\left(C_{b}, T\right)$ is presented, or any other thermal expectation value depending on $C_{b}$ and $T$. 
Along the $x$-axis for $T=0$, one must consider quantum fluctuations ("zero-point fluctuations"), which by the ellipticity of the spin precession reduce the magnetisation from the equivalent of the spin quantum number $s$ to an effective value, which is smaller:

$\left\langle\vec{k}\left|\hat{S}_{\vec{l}}^{z}\right| \vec{k}\right\rangle=s \quad \rightarrow \quad\left\langle\vec{k}\left|\hat{S}_{\vec{l}}^{z}\right| \vec{k}\right\rangle=s-\left|\gamma_{2}(\vec{k})\right|^{2}$.

Here $|\vec{k}\rangle:=\sum_{\vec{r}_{l}} \frac{\hat{S}_{\vec{l}}^{x}-\mathrm{i} \hat{S}_{l}^{y}}{\sqrt{2 s N}} \exp \left(\mathrm{i} \vec{k} \cdot \vec{r}_{\vec{l}}\right)|G S\rangle$, where $|G S\rangle$ is the (nontrivial !) ground state of the system. The vectors $\vec{l}$ count the $N$ sites $\vec{r}_{\vec{l}}$ of the lattice considered, the $\hat{S}_{\vec{l}}^{x}$ etc. are the spin operators.

By the Bogoliubov-Valatin transformation, see e.g. page 25 in Ref. [13], the ground-state $|G S\rangle$ is not identical with the 'trivial state' $|0\rangle$, where all spins are aligned in the preferred direction (i.e. $|0\rangle \hat{=}\left|m_{l}=s\right\rangle$ $\forall l$, i.e. for all sites of the lattice). Instead, $|G S\rangle$ is obtained from $|0\rangle$ by a straightforward calculation, which hides the 'ellipticy' by a mathematical formalism described in detail in the above-mentioned 'text-book reference'. In this way one derives the following identity involving the 'ellipticity factor' $f_{k}=\frac{\left(\epsilon_{a}(\vec{k})+\epsilon_{b}(\vec{k})\right) / 2}{\sqrt{\epsilon_{a}(\vec{k}) \cdot \epsilon_{b}(\vec{k})}}$ of Eqn. (4) and the 'zero-point spin reduction' $\left|\gamma_{2}(\vec{k})\right|^{2}$ :

$$
\begin{gathered}
\left|\gamma_{2}(\vec{k})\right|^{2}=\frac{1}{2} \cdot\left(f_{k}-1\right) \equiv \\
\frac{1}{2}\left(\frac{\left(2 A / M_{s}\right) k^{2}+2 \pi M_{s}+\pi M_{s} \cdot\left(k \cdot t_{h}\right) \cdot \sin ^{2} \varphi_{\vec{k}}}{\sqrt{\left(\left(2 A / M_{s}\right) k^{2}+4 \pi M_{s}\right) \cdot\left(\left(2 A / M_{s}\right) k^{2}+2 \pi M_{s} \cdot\left(k \cdot t_{h}\right) \cdot \sin ^{2} \varphi_{\vec{k}}\right)}}-1\right) .
\end{gathered}
$$

In Fig. 1 the zero-point spin reduction $\left|\gamma_{2}(k)\right|^{2}$ is plotted as a function of $k$ for $\sin ^{2} \varphi_{\vec{k}}=1$ with Permalloy material parameters (i.e. $4 \pi M_{s}=10^{4}$ Gauss, $A=1.3 \times 10^{-6} \mathrm{erg} / \mathrm{cm}$ ) and a thickness of $t_{h}=4$ $\mathrm{nm}$, as in the magnetic nanostructures studied by Höllinger et al., [15].

As can be seen from Fig. 1, $\left|\gamma_{2}((0, k, 0))\right|^{2}$ is less than $10 \%$ for $k \gtrsim 0.1(\mathrm{~nm})^{-1}$; for these $k$-values the linearized Bogoliubov-Valatin theory makes sense. Whereas for $k$-values below that value, corresponding to almost macroscopic wavelengths, there is a strong increase of the ellipticity so that for these small wavenumbers the linearization would be no longer meaningful.

But for $t_{h} \equiv 0$ one sees that for $k \cdot l_{e} \ll 1$, where $l_{e}:=\sqrt{\frac{2 A}{4 \pi M_{s}^{2}}}=\sqrt{\frac{D}{C_{b}}}$ is the above-mentioned exchange-length $(\approx 5.7 \mathrm{~nm}$ for Permalloy), a 
divergency develops for $k \rightarrow 0: \quad\left|\gamma_{2}(\vec{k})\right|^{2} \approx \frac{1}{2} \cdot f_{\vec{k}}=1 /\left(4 k \cdot l_{e}\right) \gg$ 1. This divergency has already been mentioned above, and in the present context for $T=0$ it means that for small enough values of $k \cdot l_{e}$ spatially periodic spin fluctuations of this small wavenumber $k$ cannot be treated any longer in the Bogoliubov-Valatin theory (i.e. by linearization), since e.g. the effective spin-quantum number $s^{\text {eff }}=$ $s-\left|\gamma_{2}(\vec{k})\right|^{2}$ becomes negative for these $k$. This is true although the integral $\int \mathrm{d}^{2} k\left|\gamma_{2}(\vec{k})\right|^{2}$ is finite (whereas, as already mentioned above, for $t_{h} \equiv 0$ and finite temperatures, long-range order would not at all exist, i.e. $\left.\int \mathrm{d}^{2} k \frac{\left|2 \cdot \gamma_{2}(\vec{k})\right|^{2}+1}{\exp \beta \epsilon(\vec{k})-1} \equiv \infty\right)$. In contrast, for $t_{h}>0,\left|\gamma_{2}(\vec{k})\right|^{2}$ would diverge in a similar way, namely as $\approx 0.25 / \sqrt{\left(k \cdot t_{h} / 2\right) \sin ^{2} \varphi_{\vec{k}}+k^{2} \cdot l_{e}^{2}}$, where the first term depends essentially only on the thickness and not on the other parameters, but long-range order at finite $T$ exists in this case nonetheless for all values of $C_{b}$. So the convergence of the integral for $M_{s}(T)$ and the behaviour of the zero-point reduction $\left|\gamma_{2}(\vec{k})\right|^{2}$ for small $k$ are not simply related.

But as a consequence of the preceding paragraphs, due to the magnetic dipole interaction there is automatically a $k$-dependent effective in-plane uniaxial anisotropy favouring the axis parallel to the magnetisation. This $k$-dependent effective anisotropy can be roughly obtained by replacing the effective field $2 \pi M_{s} \cdot k \cdot t_{h} \cdot \sin ^{2} \varphi_{k}$ by its angular average $2 \pi M_{s} \cdot k \cdot t_{h} \cdot 0.5$. Here the typical long-wavelength scale, e.g. the typical wavelength in the observation of magnetisation ripple phenomena, see Ref. [10], is given by the characteristic wavenumber $k=1 / l_{e}$. So we associate to the magnetostatic phenomena in thin films a $k$-dependent effective in-plane uniaxial anisotropy of the order of $C_{u}(k):=\mu_{B} \cdot \pi M_{s} \cdot k \cdot t_{h}$, i.e. we write $C_{u}(k)=\tilde{C}_{u} \cdot k$, with $\tilde{C}_{u}:=\mu_{B} \cdot \pi M_{s} \cdot t_{h}$. Here $k \lesssim 1 / l_{e}$.

In Fig. 2, again for the already mentioned Permalloy parameters, we present results for the 'naive magnetisation decay' $M_{s}(T)$, i.e. calculated according from the formula (4) for $d=2$ with the 'ellipticity factor' $f_{k}$, but without the 'interaction factor' $F_{k}(T)$. By comparison with similar results for $d=3$, where the $T^{3 / 2}$ behaviour is quite evident, we get for $d=2$ the already mentioned 'Döring behaviour', i.e. the result looks like $\left(M_{s}(T)-M_{s}\left(T_{0}\right)\right) \propto T \cdot \ln \frac{T}{T_{0}}$, where $T_{0}$ is a temperature representing an effective 'cutoff' at low k (see the figure caption 
of Fig. 2).

Since the experimental observations deviate apparently from the Döring behaviour in a significant way, the additional 'interaction factor', mentioned above, seems really necessary.

On the horizontal coordinate axis of our plot one has thus an 'ordered line segment' ranging from the above-mentioned value $C_{u}$, calculated e.g. for $k \equiv 1 / l_{e}$ or any other relevant characteristic reciprocal length of the system, up to infinity, whereas the dangerous 'infrared ellipticity fluctuations' corresponding to smaller wavenumbers are 'gapped away' at very low temperatures, since the amplitudes behave $\propto \exp \left(-\frac{C_{u}}{k_{B} T}\right)$.

From the value on the $\mathrm{x}$-axis corresponding to the above-mentioned standard case (e.g. Permalloy) on the 'ordered line segment' a flow line emerges, which is well-defined if along the third axis (' $z$ '-axis) a quantity like $M_{s}\left(C_{b}, T\right)$ is plotted, and which flows to the thermal critical point.

This line begins at $T=0$, i.e. in the quantum regime, with a positive vertical slope, but soon it may turn to the left, since with increasing $T$ the typical wavenumbers increase, the ellipticity decreases, and the exchange interactions become important. So the flow line in this region may resemble (details are not needed in the following !) a flat elliptical segment running almost parallel to the x-axis in the direction of the $\mathrm{y}$ axis, until it extrapolates, almost horizontally, to the above-mentioned small crossover temperature $T_{1}\left(\cong \sqrt{2} T_{M}\right.$, see below). This should signal the crossover from the linear rsp. square root behaviour $\epsilon(\vec{k}) \cong$ $\sqrt{C_{b} \cdot D k^{2}+\tilde{C}_{u} \cdot k}$ of the dispersion for $k \cdot l_{e} \lesssim 1$ to parabolic behaviour, $\epsilon(\vec{k}) \cong D \cdot k^{2}$ for $k \cdot l_{e} \gtrsim 1$. Therefore one roughly expects $k_{B} T_{1} \cong \sqrt{2} C_{b}$, see e.g. Eqn. (3) for $t_{h}=0$ : It is, in fact, visuable from Fig. 3, that there is a rather sharp crossover of this kind at $k_{c}:=\pi t_{h} / l_{e}^{2} \cong 0.1$ $(n m)^{-1}$. At shorter wavelengths (larger $k$ ), the exchange interaction dominates and the interaction energy is $\approx k_{B} T_{1}$ or larger.

It is not essential (and not at all universal) how this 'crossover point' is reached, i.e. from which ground state at $T=0$ (as we have seen the ground states will be nontrivial, i.e. not given by the BogoliubovValatin transformation at length scales $k \cdot l_{e} \ll 1$ ). In contrast, in the Bloch region $T_{1}<T<T_{2}$, where the dispersion of the magnons, whether interacting or not, is dominated exclusively by the exchange 
interaction, the behaviour is essentially universal, e.g. the values of the anisotropies, including the effects of the magnetostatic interactions, become irrelevant.

Analogously, at the second axis ('y'-axis) one has the following scenario: long-range magnetic order up to the thermal critical temperature (Curie temperature) $T_{c}$, which is of the order of the maximal magnon energy, $T_{\max }: \cong D \cdot\left(\frac{\pi}{a}\right)^{2} / k_{B}$, or typically even larger, [16]. From this Curie fixed point, i.e. in the thermal regime, our (backward) flow line emerges starting at first horizontally, but soon turning downwards, until it flows back from $T_{2}: \cong T_{\max } / 3$ towards $T_{1}$ essentially according to Bloch's $T^{3 / 2}$-law, thereby reaching the above-mentioned crossover point, where $k_{B} T_{1} \approx \sqrt{2} C_{b}$, as already noted. From there the (backward) flow returns to the starting-point (corresponding e.g. to ultrathin Permalloy films with finite $t_{h} \ll l_{e}$ ) on the 'ordered line segment' of the $x$-axis, where the linearized (i.e. Bogoliubov-Valatin) transformation should be valid until the very small effective in-plane uniaxial anisotropy of the order of $C_{u} \equiv C_{b} \cdot \frac{t_{h}}{l_{e}}$ : But this last part of the flow is non-universal, i.e. $C_{u}$ can also be replaced by a quantity not depending on $C_{b}$.

Now, what counts in the Bloch behaviour between $T_{1}$ and $T_{2} \cong$ $\frac{1}{3} T_{\max }$, are the thermal energy factor $\propto T$ and the effective interaction radius (in $\vec{k}$-space) $\propto T^{1 / 2}$ corresponding to only the segment of the flow line between $T_{1}$ and $T_{2}$ (in this region the quasi-classical 'poor man's scaling' considerations apply).

\section{Two-dimensional systems with positive out-of- plane anisotropy}

For two-dimensional systems with a positive uniaxial perpendicular anisotropy, i.e. favouring (in contrast to the previous case) an out-ofplane orientation, there is no ellipticity of the spin precession around the $z$-direction, and the temperature dependence of the magnetisation follows in a naive formulation analogously to Eqn. (4), namely $M_{s}(T)=M_{s}(0)-$ const. $\cdot \pi \times \int_{k=0}^{\pi / a} \frac{2 k \mathrm{~d} k}{\exp \beta \epsilon(k)-1}$, with $\epsilon(k)=C_{b}^{\text {out }}+D \cdot k^{2}$, where $C^{\text {out }}:=-C_{b}-4 \pi M_{s}>0$, whereas in-plane magnetostatic fields 
are now neglegible.

The above-mentioned integral can be evaluated analytically, yielding a variant of the 'Döring behaviour', namely

$M_{s}(T) \equiv M_{s}(0)-$ const. $\cdot \pi \times\left(\frac{k_{B} T}{D}\right) \cdot\left(-\frac{D \cdot(\pi / a)^{2}}{k_{B} T}+\ln \frac{e^{x_{\max }}-1}{e^{x_{\min }}-1}\right)$,

with $\epsilon_{\max }:=\beta \cdot\left(C_{b}^{\text {out }}+D \cdot\left(\frac{\pi}{a}\right)^{2}\right)$ and $\epsilon_{\min }:=\beta \cdot C_{b}^{\text {out }}$.

Again, if in accordance with [3] a $T^{3 / 2}$-behaviour would be observed, an additional $T^{1 / 2}$-factor would be necessary.

In the particular case of a 'reorientation transition', corresponding to a situation, where the effective uniaxial anisotropy would be zero, $C_{b}^{\text {out }} \equiv 0$, for short-range interactions the original Mermin-Wagner theorem would become applicable and the magnetic order would vanish: Instead, again by the (long-range) dipolar interactions, one observes a nontrivial domain structure.

At this place it should be be mentioned that the 'reorientation transition', and in particular spinwave excitations (including the dipolar interactions and surface anisotropies) in systems with a finite number of non-equivalent magnetic planes with different tilt angles of the magnetization direction in these planes, have been carefully studied in a series of papers by Erickson and Mills, [20, 21, 22].

\section{$6 \quad \mathbf{d}=1: T^{5 / 2}$-behaviour ?}

Here we consider a homogeneous ferromagnetic wire stretching from $x=-\infty$ to $x=+\infty$ along the $x$-axis, with constant circular cross section, magnetised longitudinally, i.e. in the x-direction. Now the dipolar (effective) anisotropy acts with equal strength, $C_{b}=\mu_{B} \cdot 2 \pi M_{s}$, along the $y$-axis and the $z$-axis, so that, by applying the 'naive approach' of Eqn. (4) again, we get (almost as before) $M_{s}(T)=M_{s}(0)-$ const. $\times$ $\int_{0}^{\pi / a} \frac{\mathrm{d} k}{\exp \beta \cdot \epsilon(\vec{k})-1}$, with $\epsilon_{k}=\mu_{B} \cdot 2 \pi M_{s}+D \cdot k^{2}$. For temperatures allowing the approximation $\exp (\beta \epsilon(\vec{k}))-1 \cong \beta \epsilon(\vec{k})$ this can be directly evaluated and yields $M_{s}(T)=M_{s}(0)$ - const. $\times T \times \pi / 2 \sqrt{\mu_{B} \cdot 4 \pi A}$, whereas for general temperatures one gets the numerical behaviour presented in Fig. 4, i.e. also a behaviour $\propto T$. 
That this behaviour cannot be valid to arbitrary low temperatures follows from the Maxwellian relation $\partial S / \partial H=\partial M / \partial T$ and Nernst's 'Third Fundamental Theorem' of thermodynamics, according to which $\partial S / \partial H \rightarrow 0$ for $T \rightarrow 0$.

In fact, experimentally one has obtained instead, see [3], a pronounced $T^{5 / 2}$-dependence, in agrrement with the apparently universal classification of U. Köbler for half-integer $s$ and one-dimensional systems, [3], and astonishingly also with the experiments by U. Gradmann and coworkers, [23], on Fe monolayers on W(110) (covered by Ag). Also the films with the different $T^{3 / 2}$-behaviour studied in [5] are planar Fe films on W(110), but thicker (from 3 to 21 monolayers), and whereas (as already mentioned above) in [5] one has the $T^{3 / 2}$ behaviour discussed in the two preceding sections, for the films of [23] one has instead the $T^{5 / 2}$ behaviour, which is still more astonishing. Not only for this system, but also for $d=1$ this is unexpected and suggests that here the 'interaction damage' factor $F_{k}(T)$ is even stronger, namely $\propto T^{3 / 2}$ instead of $\propto T^{1 / 2}$. Presently, this is not at all understood, or only very qualitatively: One knows that in one dimension the critical thermal fluctuations destroy long range order at finite $T$ even for Ising models, whereas in $d=2$ the fluctuations are substantial for $X Y$-models, but not for Ising models. So it is not quite surprising that already for the Bloch region the interaction factor should be much stronger for $d=1$ than for $d=2$.

Topological excitations may play an essential role also in this case, namely the so-called Bloch-point excitations, which are studied in a recent paper of Thiaville et. al., [24]. These are very 'floppy'; they consist topologically of opposite spin directions along a certain axis, e.g. $\vec{S} \propto \pm \hat{z}$ for $z>0$ rsp. $z<0$, but in the $x y$-plane there can be a vortex. In this case, the 'damage factor' $F_{k}(T)$ corresponding to vortex interactions exists for all three orthogonal planes, the $x y$-plane, the $y z$-plane and the $z x$-plane.

In any case, the $T^{5 / 2}$-behaviour in the Bloch region does not contradict the fact that ultimately, i.e. after a further crossover to the genuine thermal critical region, there can exist not even Ising critical order in the wire, such that finally $T_{c} \rightarrow 0$. For Gradmann's monolayer films, [23], instead, after the pseudo-one-dimensional (non-Ising) behaviour in the Bloch region, the system should return to the genuine 
two-dimensional critical (Ising-) behaviour near $T_{c}$.

So this is quite complicated and subtle, as usual if one is dealing with dipolar interactions.

\section{$7 \quad T^{2}$ crossover-scaling for itinerant magnets in $\mathbf{d}=3$}

The arguments of the following section look especially simple (although this may be deceptive): We consider a crystalline metallic ferromagnet in $\mathrm{d}=3$ dimensions. The system has separate energy bands $\left\{E_{\uparrow}(\vec{k})\right\}$ rsp. $\left\{E_{\downarrow}(\vec{k})\right\}$, and at $T=0$ the 'Stoner excitations', i.e. electron-hole excitations with spin flip, where e.g. an electron with energy 'immediately below the Fermi energy $E_{F}$ ', with initial-state wave-vector $\vec{k}$ and spin $\uparrow$, is moved to a state 'immediately above $E_{F}$ ', with a different final-state wave-vector $\vec{k}^{\prime}$ and spin $\downarrow$. Now at finite $T$, the expression 'immediately above or below $E_{F}$ ' means: 'within an interval of width $\approx k_{B} T$ around $E_{F}$. Every such excitation reduces the magnetic moment of the sample by two Bohr magnetons; i.e. in the above-mentioned 'heuristical formula' one has a 'phase-space factor' $\propto\left(k_{B} T\right)^{2}$, where one of the two powers of $k_{B} T$ stands for the effective phase-space of the $i n i$ tial states, while the other one represents the final states. There is no 'energy factor', since all this happens in the above-mentioned interval immediately near $E_{F}$.

Similar arguments also apply to itinerant antiferromagnets, in accordance with the observations of U. Köbler, [3], who always observed the same Bloch exponent for the magnetisation of ferromagnets rsp. the sublattice magnetisation of antiferromagnets (As a consequence the Bloch scaling is probably not directly related to quantum-criticality, since the quantum critical behaviour of ferromagnetic and antiferromagnetic systems is different). In contrast, for semiconducting systems as EuO and EuS the magnetism should better be described by a (non-itinerant) $d=3$ Heisenberg model with $s=7 / 2$, and so one expects the $T^{3 / 2}$-behaviour for $d=3$. But an 'interaction factor' of $T^{1 / 2}$ would transform this into the observed $T^{2}$ behaviour, in accordance with [3]. Yet such a behaviour would definitely go beyond the Heisenberg model, since within that model there is the rigorous analysis of Dyson, Ref. [4], for arbitry fixed spin quantum number $s$, see Ref. [4], predicting a more gradual decrease: $M_{s}(T)=$ 
$M_{s}(0)-c_{1} \cdot T^{3 / 2}-c_{2} \cdot T^{5 / 2}-c_{3} \cdot T^{7 / 2}-c_{4} \cdot T^{4}-\mathcal{O}\left(T^{9 / 2}\right)$. Note that the first term with integer exponent is $\propto T^{4}$, and not $\propto T^{3}$; the integer term results mainly from the decrease of the magnon stiffness, which according to rigorous theories 'a la Dyson' should be $\propto T^{5 / 2}$, whereas more phenomenological theories, and according to Köbler, Ref. [3], also the experiments, suggest a decrease following an effective behaviour as $D(T)=D(0)-$ const. $\cdot T^{3 / 2}$.

Because of the large value of $s=7 / 2$ for $\mathrm{Eu}^{2+}$, the 'non-Dysonian' behaviour, and the 'extra' interaction factor $\propto T^{1 / 2}$, look not completely unreasonable. And one should note again that this behaviour goes definitely beyond the (underlying) Heisenberg model, which always assumes fixed $s$, in contrast to the Pippard arguments from the previous section and those discussed below in connection with the problem of the difference between integer and half-integer $s$. And one should also note, concerning the case of metallic magnetism, that the Stoner mechanism (which is actually a two-particle mechanism) looks completely different from anything formulated in the Heisenberg model. In contrast, the collective magnon excitations, which are also present in itinerant magnets and which have the same quadratic dispersion as before, seem to give a neglegible contribution to the behaviour of $M_{s}(T)$ in the temperature range considered, although at very low temperatures a $T^{3 / 2}$-contribution would always be larger than a $T^{2}$-one. So this means again that one should remain above a certain crossover-temperature, in this time that one to the dominance of the $T^{3 / 2}$ behaviour for $d=3$.

\section{Integer $s$}

The arguments in the previous sections have all considered half-integer values of $s$, e.g. $s=1 / 2$ and $s=7 / 2$. For integer values, e.g. $s=1$, one must consider the additional interaction energy, $\sim k_{B} T$, which is necessary to break e.g. a $s=1$ triplet state of two $s=1 / 2$ spins into two separate $s=1 / 2$ entities. This happens at sufficiently high temperatures at the upper range of the Bloch region, i.e. below the genuine critical temperature region.

So for a single entity the square root of that energy comes into play as an additional energy factor so that Köbler's effective Bloch exponents of $\epsilon_{B}=3 / 2$ (rsp. $\left.\epsilon_{B}=5 / 2\right)$ for spin systems with half-integer 
$s$ and (effective) Bloch-dimensionality $d=2$ (rsp. $d=1$ ) should change for integer $s$ to $\epsilon_{B}=4 / 2$ (rsp. $\epsilon_{B}=6 / 2$ ), apparently as observed, see [3]. For $d=3$ things are more complicated: according to the tentative arguments of the previous section, the Bloch exponent $\epsilon_{B}=2$ for $d=3$ and half-integer $s$ is given by a product of three terms, which can be written symbolically as $\left(k_{B} T\right)_{\text {initial state }}^{1} \times\left(k_{B} T\right)_{\text {final state }}^{1} \times$ $\left(k_{B} T\right)^{0}$, where the third term represents the constant energy-factor. However for integer $s$, due to the above-mentioned interaction effects, the first two factors should each acquire an additional power of $k_{B} T$, while the third factor should acquire an additional 'square-root power' $\left(k_{B} T\right)^{1 / 2}$. In this way, starting with the $T^{2}$-behaviour for half-integer $s$ one would expect a $T^{9 / 2}$-behaviour for integer $s$, as observed, [3]. Whether this is more than heuristics, has to be shown, similarly to the previous sections, but it should be noted that the pronounced crossover from a $T^{2}$ low-temperature behaviour to a $T^{9 / 2}$ behaviour at higher temperatures, which U. Köbler observes for $\mathrm{NiO}$ at $\mathrm{T}=100 \mathrm{~K}$ rsp. for $F e$ at $628 \mathrm{~K}$, seems to be in favour of the present scenario ${ }^{2}$.

\section{Conclusions}

As a consequence of the preceding sections, concerning the temperature range considered, it should be noted that the arguments of the present paper, supported by quantitative calculations especially in sections 2 and 3, do neither concentrate on the magnetostatic interactions (although these are very important, as seen in those chapters) nor stress the immediate vicinity of the quantum region at $T=0$ nor that of the thermal critical region near $T_{c}$, but rather concentrate on the (wide) 'Bloch temperature range' between the (rather low) crossover temperature $T_{1}\left(\cong \sqrt{2} C_{b} / k_{B}\right)$ and a (much higher) crossover temperature of the order of magnitude of $T_{2} \cong \frac{1}{3} D \cdot(\pi / a)^{2} / k_{B}$.

So quantum behaviour is significant, but at the same time, in the Bloch region, it only plays a secondary role, apparently in contrast to interactions, which appear to be particularly important for $d=2$, where they are known to destroy the long-range order for the Kosterlitz-

\footnotetext{
${ }^{2}$ At several places in the observations of U. Köbler, see [3], also a crossover from a $T^{3 / 2}$ behaviour 'at low temperatures', e.g. for $T \lesssim 200 \mathrm{~K}$, to a $T^{2}$-behaviour at a considerable range of higher temperatures is observed.
} 
Thouless phase transition. In higher dimensions also the main implicit assumption of the Heisenberg model, that the spin quantum-number $s$ is invariably constant, becomes questionable through the interactions already in the Bloch region.

All statements in the previous sections, except from section 8, apply to half-integer $s$ in Köbler's classification, [3]. Already at this place, i.e. for half-integer s, the role of the interactions seems crucial, particularly for $d=2$ and $d=1$. Additional complications for integer $s$ (outside the region of overwhelming importance of the thermal fluctuations) have been discussed in section 8 .

A more thorough analysis of the questions left open, which mainly concern the role of interactions, is shifted to future work.

\section{Acknowledgements}

The author acknowledges stimulating communications from K. Binder, P. Bruno (who pointed out an error in an earlier version of this paper), U. Gradmann, U. Köbler and S. Krompiewski, and fruitful discussions with C. Back, G. Bayreuther, R. Höllinger, A. Killinger, W. Kipferl, and J. Siewert.

\section{References}

[1] W. Kipferl, Spin-wave excitations in epitaxial Fe(001)-nanostructures on GaAs, unpublished seminar, University of Regensburg, October 2002; W. Kipferl, M. Dumm, M, Rahm, G. Bayreuther, J. Appl. Phys. 93 (2003), in press.

[2] F. Bloch, Z. Physik 61 (1930) 206

[3] U. Köbler, e.g. J. Phys.: CM 14 (2002) 8861, and references cited therein.

[4] F.J. Dyson, Phys. Rev. 102 (1956) 1230

[5] J. Korecki, M. Przbylski, U. Gradmann, J. Magn. Mater Magn. Mater. 89 (1990) 325; see also Fig. 5 in [3] 
[6] N.D. Mermin, H. Wagner, Phys. Rev. Lett. 17 (1966) 1133 ; the Mermin-Wagner theorem states that in the Heisenberg or XYmodels with short-range interactions and for fixed spin quantum number $s$ there cannot be magnetic long-range order in $\mathrm{d}=2$ dimensions.

[7] P.W. Anderson, A poor man's derivation of scaling laws ..., J. Phys. C: Solid St. Phys. 3 (1970)

[8] P. Bruno, Phys. Rev. Lett. 87 (2001) 137203

[9] J.M. Kosterlitz, D.J. Thouless, J. Phys. C: Solid St. Phys. 6 (1973) 1181

[10] H. Hoffmann, J. Appl. Phys. 35 (1964) 1970

[11] U. Krey, Phys. kondens. Materie 6 (1967) 218 (in German)

[12] A.N. Slavin, S.O. Demokritov, B. Hillebrands, in: Spin Dynamics in Confined Magnetic Structures I (B. Hillebrands, K. Ounadjela, Eds.), Springer-Verlag, Berlin-Heidelberg-New York (2001), p. 35, eqs. (11)-(14)

[13] Ch. Kittel, Quantum Theory of Solids, J. Wiley, New York (1963), p. 25

[14] S.V. Maleev, Zh. Eksp. Teor. Fiz. 70 (1976) 2374 [Sov. Phys.JETP 43 (1976) 1240]; in this paper the low-temperature $T^{3 / 2}$-behaviour of the magnetisation is mentioned, but without derivation.

[15] In a magnetic vortex structure of a flat circular nano-dot the vortex core has a radius, which is of the order of $l_{e}$, see R. Höllinger, A. Killinger, U. Krey, J. Magn. Magn. Mater. 261 (2003) 178

[16] The Curie temperature of a Heisenberg model with a large value of $s$ is $\propto s^{2}$, whereas the largest magnon energy is only $\propto s$. Here we only need $T_{1} \ll T_{2}$, where $T_{2}$ (rsp. $T_{1}$ ) is the upper (rsp. lower) crossover temperature of the 'Bloch region' $T_{1}<T<T_{2}$.

[17] W. Döring, Z. Naturforsch. 16a (1961), 1008 (in German)

[18] P. Bruno, Phys. Rev. B 43 (1991) 6015 
[19] J.M. Kosterlitz, J. Pys. C: Solid State Phys. 7 (1974) 1046

[20] R.P. Erickson, D.L. Mills, Phys. Rev. B 43 (1991) 10715

[21] R.P. Erickson, D.L. Mills, Phys. Rev. B 44 (1991) 11825

[22] R.P. Erickson, D.L. Mills, Phys. Rev. B 46 (1992)

[23] M. Przybilski, U. Gradmann, Phys. Rev. Lett. 59 (1987) 1152;

Fig. 3 is re-analyzed as Fig. 8 in [3]

[24] A. Thiaville, J.M. Garcia, R. Dittrich, J. Miltat, T. Schrefl, Phys. Rev. B 67 (2003) 094410 


\section{$\underline{\text { Figures and Figure Captions }}$}

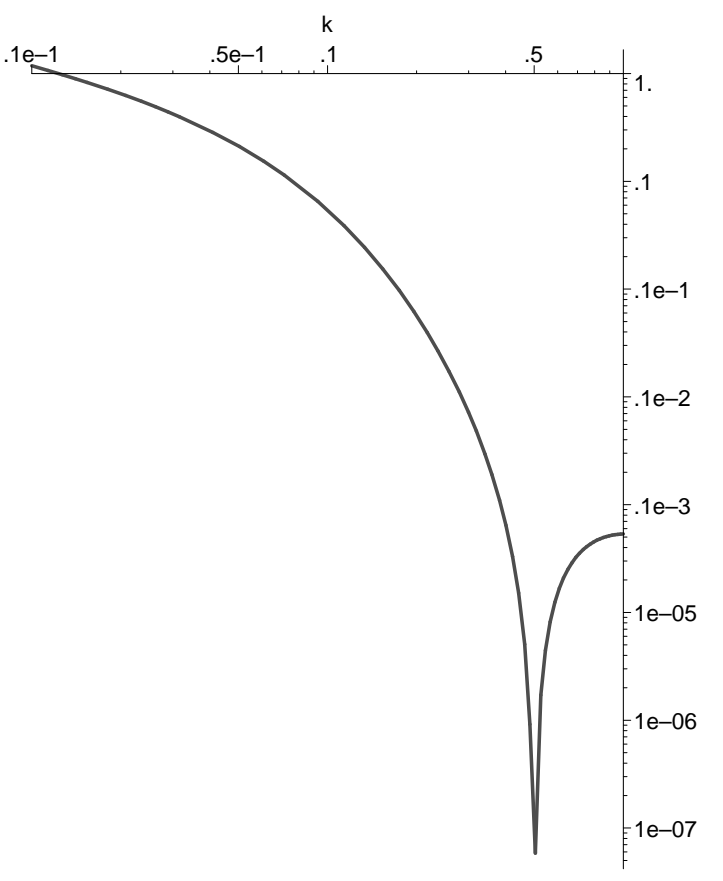

Fig.1: The zero-point spin reduction $\left|\gamma_{2}(\vec{k})\right|^{2}$ (see the text, especially Eqn. (3)) is presented over the wavevector $\vec{k}=(0, k, 0)$, where $k$ is measured in units of $(\mathrm{nm})^{-1}$, for an ultrathin Permalloy film of thickness $t_{h}=4 \mathrm{~nm}$, in a double-logarithmic plot with material parameters $4 \pi M_{s}=10^{4}$ Gauss, where $M_{s}$ is the cgs-magnetisation, and $A=1.3 \times 10^{-6} \mathrm{erg} / \mathrm{cm}$ the spin-wave stiffness.

The characteristic magnetostatic exchange length $l_{e}=\left(2 A /\left(4 \pi M_{s}\right)\right)^{1 / 2}$ is $5.7 \mathrm{~nm}$, such that the characteristic value $k_{c}:=t_{h} /\left(2 l_{e}^{2}\right)$ is $\approx 0.06$ $(\mathrm{nm})^{-1} ;\left|\gamma_{2}(\vec{k})\right|^{2}$ vanishes at $k=\pi / l_{e}$ i.e. at $\approx 0.5(n m)^{-1}$. For $k \gtrsim 0.1(\mathrm{~nm})^{-1}$ it is less than $10 \%$. For these $k$-values the linearized Bogoliubov-Valatin theory makes sense. Whereas for $k$-values below that value, corresponding to almost macroscopic wavelengths, there is a strong increase of the ellipticity so that for these small wavenumbers the linearization would be no longer meaningful. 


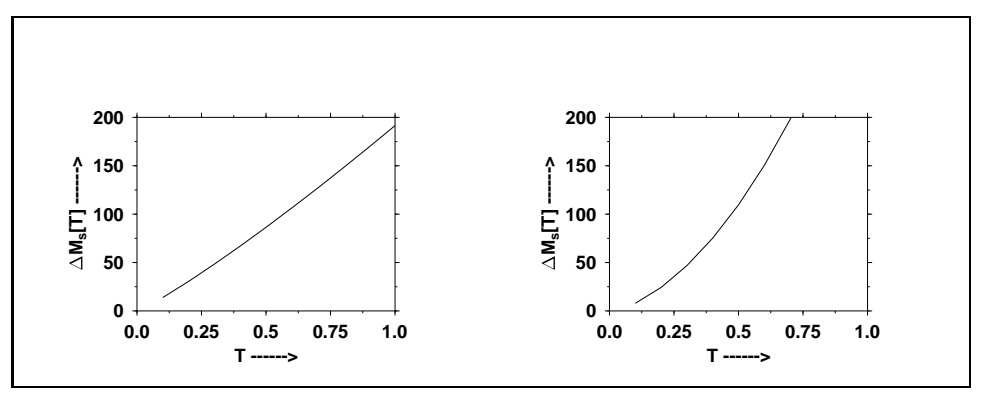

Fig. 2: Here for dimensionalities $d=2$ (left panel) and $d=3$ (right panel), respectively, the integral $\Delta M_{s}(T):=M_{s}(0)-M_{s}(T) \propto$

$$
\begin{aligned}
& \int_{k=0}^{k=\pi} \frac{\mathrm{d}^{d} k}{\exp \left\{T^{-1} \sqrt{\left.\left[2 \cdot(1-\cos k)+C_{b}\right] \cdot\left[2 \cdot(1-\cos k)+k \cdot \tilde{C}_{u} \cdot \frac{2 k_{y}^{2}}{k^{2}}\right]\right\}-1}\right.} \\
& \times \frac{2 \cdot(1-\cos k)+\frac{C_{B}}{2}+k \cdot \tilde{C}_{u} \cdot \frac{k_{y}^{2}}{k^{2}}}{\sqrt{\left[2 \cdot(1-\cos k)+C_{b}\right] \cdot\left[2 \cdot(1-\cos k)+k \cdot \tilde{C}_{u} \cdot \frac{2 k_{y}^{2}}{k^{2}}\right]}}
\end{aligned}
$$

is presented over the reduced Kelvin temperature $T$.

This plot represents schematically (in the left panel) the temperature dependence of the magnetisation of an in-plane magnetised ultrathin film under the influence of a typical exchange interaction $\epsilon=$ $2 \cdot(1-\cos k)$, which behaves for small $k$ as $\epsilon=k^{2}$, while the reduced dipolar anisotropy $\propto 4 \pi M_{s}$ is represented by $C_{b}=0.01$, and, finally, a $k$-dependent effective in-plane uniaxial anisotropy corresponding to the last term in Eqn. $(3), \epsilon(k) \propto \sqrt{\ldots+\left(4 \pi M_{s}\right)^{2} \cdot\left(k \cdot t_{h} / 2\right) \cdot\left(k_{y}^{2} / k^{2}\right)}$, is represented by the terms $\propto k \cdot \tilde{C}_{u}$, where $\tilde{C}_{u}=0.001$ is chosen. The results of the left panel correspond roughly to the 'Döring behaviour' $70 \cdot T \cdot \ln \frac{T}{0.05}$, which necessitates for $d=2$ an additional interaction factor $F_{k}(T) \propto T^{1 / 2}$ to obtain a $T^{3 / 2}$-behaviour, whereas the ' $d=3$ results' of the right panel (from which the difference can be seen) would yield just this behaviour. 


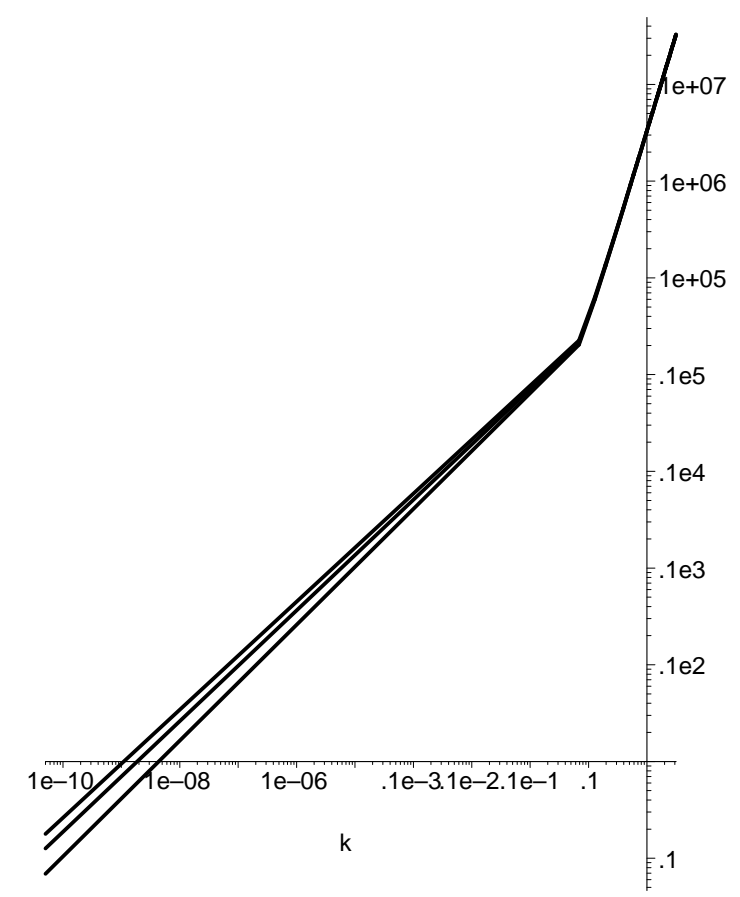

Fig. 3: In a double-logarithmic plot the energy $\epsilon(\vec{k})$ (up to a constant factor) is presented over $k$ for $\vec{k}=(0, k, 0)$, for material parameters of permalloy as in the preceding figure, where $k$ is measured in $(n m)^{-1}$. The thicknesses of the ultrathin films considered, are $4 \mathrm{~nm}, 2 \mathrm{~nm}$, and $0.6 \mathrm{~nm}$, respectively, corresponding to the highest, medium rsp. the lowest curve.

Note the sharp crossover around $k=k_{c} \equiv \pi t_{h} / l_{e}^{2} \cong 0.1 \mathrm{~nm}^{-1}$. For shorter wavelengths (larger $k$ ) the different thicknesses can no longer be distinguished, since the exchange interaction dominates. 


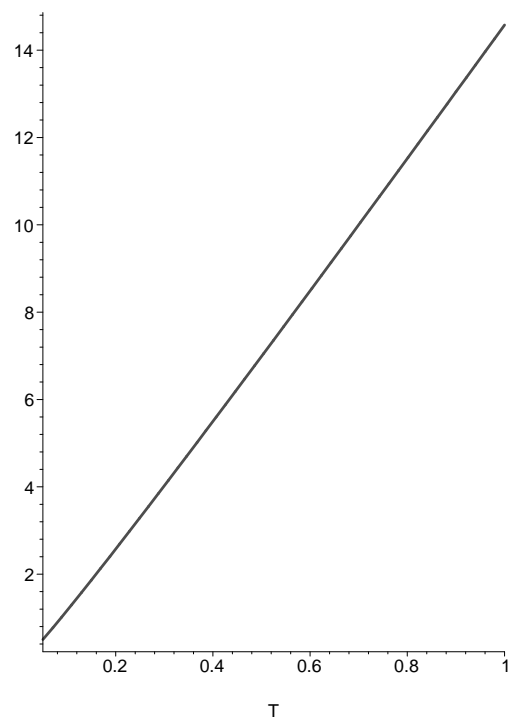

Fig. 4: For $C_{b}=0.01$, as in Fig.2, the integral $M_{s}(0)-M_{s}(T) \propto$

$$
\int_{k=0}^{k=\pi} \frac{\mathrm{d} k}{\exp \left\{T^{-1}\left[2 \cdot(1-\cos k)+C_{b}\right]-1\right\}}
$$

is presented over the reduced Kelvin temperature $T$. The results should be valid for $d=1$. 